\title{
Symmetric Extensions of Quantum States and Local Hidden Variable Theories
}

\author{
Barbara M. Terhal, ${ }^{1,2}$ Andrew C. Doherty, ${ }^{2}$ and David Schwab ${ }^{3}$ \\ ${ }^{1}$ IBM Watson Research Center, P.O. Box 218, Yorktown Heights, New York 10598 \\ ${ }^{2}$ Institute for Quantum Information, Caltech 107-81, Pasadena, California 91125 \\ ${ }^{3}$ Department of Physics, Cornell University, 109 Clark Hall, Ithaca, New York 14853
}

(Received 18 October 2002; published 16 April 2003)

\begin{abstract}
While all bipartite pure entangled states violate some Bell inequality, the relationship between entanglement and nonlocality for mixed quantum states is not well understood. We introduce a simple and efficient algorithmic approach for the problem of constructing local hidden variable theories for quantum states. The method is based on constructing a so-called symmetric quasiextension of the quantum state that gives rise to a local hidden variable model with a certain number of settings for the observers Alice and Bob.
\end{abstract}

DOI: 10.1103/PhysRevLett.90.157903

PACS numbers: 03.65.Ud, 03.67.Mn

It was Bell [1] who quantified how measurements on entangled quantum mechanical systems can invalidate local classical models of reality. His original inequality has generated a field of research devoted to general Bell inequalities and experimentally observed violations of such inequalities.

Perhaps surprisingly, the nature of the set of states that violate local realism is poorly understood, although it is known from the seminal work of Werner [2] that not all entangled states violate a Bell inequality. Recent results in quantum information theory have revealed the complex structure of the set of entangled states but have as yet shed little light on the relation between this structure and violation of Bell inequalities. For example, it has been conjectured by Peres [3] that so-called bound entangled states which satisfy the Peres-Horodecki "partial transposition" criterion [4] (i.e., they have positive partial transpose), do not violate any Bell inequalities. There are various results that support this conjecture both in the bipartite and multipartite case, see Ref. [5], but none of the results is conclusive.

What has been lacking in the literature so far is a systematic way of deciding whether a quantum state does or does not violate some Bell inequality. The difficulty is that the possible types of local measurements and the number of measurements that observers can perform is in principle unbounded and the enumeration of Bell inequalities is computationally hard [6].

In this Letter we present the first systematic approach for constructing local hidden variable theories for quantum states, depending only on the number of local measurement settings for each observer. Our approach has yielded both numerically constructed local hidden variable theories for a variety of quantum states as well as analytical results for Werner states [2] and a class of bound entangled states based on real UPBs [7].

Before we can state our main result, we recapitulate the mathematics of local hidden variable (LHV) models and Bell inequalities for bipartite systems. We refer the reader to Refs. $[3,6,8]$ for some literature on the theoretical formulation of general Bell inequalities. Each of the observers, Alice and Bob, has a set of local measurements. Let $i=1, \ldots, s_{a}$ be the number of measurements for Alice and let each measurement have $o_{a}(i)$ outcomes. Let $k=1, \ldots, s_{b}$ be the number of measurements for Bob and $o_{b}(k)$ be the number of outcomes per measurement. The probability $P_{i j, k l}$ denotes the probability that Alice's $i$ th measurement has outcome $j$ and Bob's $k$ th measurement has outcome $l$. A local hidden variable model assumes the existence of a shared random variable between Alice and Bob that is used to locally generate a measurement outcome depending only on the choice of the local measurement (and not on the choice of the other, remote, measurement). The local hidden variable model generates the probability vector $\vec{P}$ with entries $P_{i j, k l}$ when it generates measurement outcomes in accordance with these probabilities. Mathematically one defines a convex set $S\left(s_{a}, s_{b}, o_{a}, o_{b}\right)$ which is the set of probability vectors $\vec{P}$ that can be generated by LHV models. It is known that $S$ is a polytope and that the extremal vectors $\vec{B}$ of $S$ are vectors with 0, 1 entries [8]. These extremal vectors $\vec{B}$ correspond to the situation in which the outcomes of the measurements are determined with certainty and can be labeled by two sets of indices $\mathbf{m}=\left(m_{1}, \ldots, m_{s_{a}}\right)$ where $m_{i}=1, \ldots, o_{a}(i)$ and $\mathbf{n}=\left(n_{1}, \ldots, n_{s_{b}}\right)$ where $n_{k}=1, \ldots, o_{b}(k)$. A brief expression for these extremal vectors is

$$
B_{i j, k l}^{\mathbf{m}, \mathbf{n}}=\delta_{j m_{i}} \delta_{l n_{k}} .
$$

In words, each extremal vector specifies a single outcome with probability one for each local measurement, independently of the measurement made by the other parties.

For a quantum mechanical system $\rho$ in $\mathcal{H}_{d_{A}} \otimes \mathcal{H}_{d_{B}}$ the probability $P_{i j, k l}$ is given by $P_{i j, k l}(\rho)=\operatorname{Tr} E_{i j}^{A} \otimes E_{k l}^{B} \rho$. Here $\left\{E_{i j}^{A} \geq 0: \sum_{j} E_{i j}^{A}=I_{d_{A}}\right\}$ are the POVM (positive operator-valued measure) elements for Alice's $i$ th measurement and $\left\{E_{k l}^{B}\right\}$ are the POVM elements for Bob's $k$ th 
measurement. There is a violation of a Bell inequality if and only if $P_{i j, k l}$ cannot be generated by a LHV model, or $\vec{P} \notin S$.

In this Letter we prove the first necessary condition for a state to violate a Bell inequality depending only on the number of settings for Alice and Bob. We explicitly construct a LHV model in a $s_{a}=2$ and arbitrary $s_{b}$ setting (and vice versa) for any bound entangled state based on a real unextendible product basis (UPB) [4]. Then we discuss numerical work that shows that many of the known bipartite bound entangled states cannot violate a Bell inequality with two settings either for Alice or Bob. Finally, we partially reproduce and extend some of Werner's original results by showing that it is possible to use our procedure to analytically construct LHV theories for Werner states. It is noteworthy to mention that our methods (Theorem 1 and Theorem 2) straightforwardly generalize to multipartite states, even though we have not explored this direction.

We connect violations of Bell inequalities to the existence of a symmetric (quasi-) extension of a quantum state [9]. An extension of a quantum state $\rho$ on, say, a system $A B$, is another quantum state defined on a system $A B C$ such that when we trace over $C$ we obtain the original quantum state $\rho$. We are interested in the situation where the system $C=A^{\otimes\left(s_{a}-1\right)} \otimes B^{\otimes\left(s_{b}-1\right)}$ and we demand that the extension be invariant under all permutations of the $s_{a}$ copies of system $A$ among each other and similarly invariant under any permutation of the $B$ systems. It is clear that if the quantum state $\rho$ is separable, i.e., $\rho=\sum_{i} p_{i}\left(\left|\psi_{i}\right\rangle\left\langle\psi_{i}\right|\right)_{A} \otimes\left(\left|\phi_{i}\right\rangle\left\langle\phi_{i}\right|\right)_{B}$, such an extension always exists: we just copy the individual product states onto the other spaces:

$$
\rho_{\mathrm{ext}}=\sum_{i} p_{i}\left(\left|\psi_{i}\right\rangle\left\langle\psi_{i}\right|\right)^{\otimes s_{a}} \otimes\left(\left|\phi_{i}\right\rangle\left\langle\phi_{i}\right|\right)^{\otimes s_{b}} .
$$

If the state $\rho$ is a pure entangled state, then it is also clear that such a symmetric extension cannot exist. The symmetry requirement implies that the pure entangled state $\rho_{A B}$ must equal $\rho_{A^{\prime} B}$, where $A^{\prime}$ is another $A$ system, which is impossible. In popular terms we may say that pure entanglement is "monogamous": $B$ cannot be entangled with $A$ and $A^{\prime}$ at the same time. In some sense what we show in this Letter is that (i) a violation of a Bell inequality indicates that the entanglement in the quantum state is monogamous and (ii) there are many mixed entangled states whose entanglement is not monogamous.

Thus the existence of a symmetric extension can be viewed as a separability criterion (see Ref. [11] for a similar but stronger separability criterion where one demands that the symmetric extension has positive partial transposes). For considering Bell inequality violations we generalize our criterion slightly and ask whether a state has a symmetric quasiextension $H_{\rho}$ which is not necessarily positive. In order to define this notion we need the definition of a multipartite entanglement witness, which is an entanglement witness which can detect any multipartite entanglement in a state. It has the property that for all states $\psi_{1}, \ldots, \psi_{s_{a}}, \phi_{1}, \ldots, \phi_{s_{b}},\left\langle\psi_{1}, \ldots, \psi_{s_{a}}\right.$, $\left.\phi_{1}, \ldots, \phi_{s_{b}}\left|H_{\rho}\right| \psi_{1}, \ldots, \psi_{s_{a}}, \phi_{1}, \ldots, \phi_{s_{b}}\right\rangle \geq 0$.

Definition (symmetric quasiextension): Let $\pi$ : $\mathcal{H}^{\otimes s} \rightarrow \mathcal{H}^{\otimes s}$ be a permutation of spaces $\mathcal{H}$ in $\mathcal{H}^{\otimes s}$. We define

$$
\operatorname{Sym}(\rho)=\frac{1}{s !} \sum_{\pi} \pi \rho \pi^{\dagger} .
$$

We, say, that $\rho$ on $\mathcal{H}_{A} \otimes \mathcal{H}_{B}$ has a $\left(s_{a}, s_{b}\right)$-symmetric quasiextension when there exists a multipartite entanglement witness $H_{\rho} \quad$ on $\mathcal{H}_{A}^{\otimes s_{a}} \otimes \mathcal{H}_{B}^{\otimes S_{b}}$ such that $\operatorname{Tr}_{\mathcal{H}_{A}^{\otimes\left(s_{a}-1\right)}, \mathcal{H}_{B}^{\otimes\left(s_{b}-1\right)}} H_{\rho}=\rho$ and $H_{\rho}=\operatorname{Sym}_{A} \otimes \operatorname{Sym}_{B}\left(H_{\rho}\right)$.

The reason for considering such quasiextensions is clear from the following theorems which are the main results of this Letter.

Theorem 1: If $\rho$ has a $\left(s_{a}, s_{b}\right)$-symmetric quasiextension, then $\rho$ does not violate a Bell inequality with $\left(s_{a}, s_{b}\right)$ settings.

Before proving this theorem, it is important to note the generality of the result; it holds for all possible choices of measurements which includes POVM measurements with an unbounded number of measurement outcomes. We show below that the quasiextension of $\rho$ effectively creates a LHV model for $\rho$ when Alice and Bob have $s_{a}$ and $s_{b}$ arbitrary measurements.

Proof: We prove our theorem by extracting a LHV model from the quasiextension. The LHV model for $\rho$ for $\left(s_{a}, s_{b}\right)$ settings should reproduce the vector $P_{i j, k l}(\rho)=$ $\operatorname{Tr} E_{i j}^{A} \otimes E_{k l}^{B} \rho$ for all possible choices of POVM measurements $\left\{E_{i j}^{A}, E_{k l}^{B}\right\}$, as a convex combination of the extremal $B$ vectors, i.e.,

$$
P_{i j, k l}(\rho)=\sum_{\mathbf{m}, \mathbf{n}} p_{\mathbf{m}, \mathbf{n}}\left(\left\{E_{i j}^{A}, E_{k l}^{B}\right\}, \rho\right) B_{i j, k l}^{\mathbf{m}, \mathbf{n}},
$$

where $p_{\mathbf{m}, \mathbf{n}}() \geq$.0 . If a symmetric quasiextension exists for $\rho$, then $\operatorname{Tr} E_{i j}^{A} \otimes E_{k l}^{B} \rho=\operatorname{Tr}\left(E_{i j}^{A} \otimes E_{k l}^{B} \otimes \mathbf{I}\right) H_{\rho}$. Using the definition of the $B$ vectors, the properties of the POVMs $\left(\sum_{j} E_{i j}^{A, B}=I_{d_{A, B}}\right)$, and the symmetry properties of $H_{\rho}$ it is not hard to verify that

$$
P_{i j, k l}(\rho)=\operatorname{Tr} E_{i j}^{A} \otimes E_{k l}^{B} \rho=\sum_{\mathbf{m}, \mathbf{n}}\left(\operatorname{Tr} \mathbf{E}_{\mathbf{m}}^{A} \otimes \mathbf{E}_{\mathbf{n}}^{B} H_{\rho}\right) B_{i j, k l}^{\mathbf{m}, \mathbf{n}} .
$$

Here $\mathbf{E}_{\mathbf{m}}^{A}=E_{1 m_{1}}^{A} \otimes E_{2 m_{2}}^{A} \otimes \ldots \otimes E_{s_{a} m_{s_{a}}}^{A}$ and similarly for $\mathbf{E}_{\mathbf{n}}^{B}$. Since $H_{\rho}$ is a quasiextension $p_{\mathbf{m}, \mathbf{n}}\left(\left\{E_{i j}^{A}, E_{k l}^{B}\right\}, \rho\right) \equiv \operatorname{Tr} \mathbf{E}_{\mathbf{m}}^{A} \otimes$ $\mathbf{E}_{\mathbf{n}}^{B} H_{\rho} \geq 0$, and we have obtained a LHV model.

One way of looking at this result is the following [12]. If $\rho$ has a symmetric extension $\tilde{\rho}$, then instead of measurement on $\rho$, Alice and Bob can do measurements on $\tilde{\rho}$. Because of the symmetry Alice can do the first measurement on the first Alice space and the second measurement on the second Alice space, etc. But now these measurements are all commuting and can be considered as one big 
measurement. But we know that when Alice and Bob each have only a single measurement a LHV model for their measurements exists and thus we have a LHV model for the measurements on $\rho$. With this picture in mind, it is not hard to understand the following strengthening of our results (see also Ref. [10]):

Theorem 2: If $\rho$ has a $\left(1, s_{b}\right)$-symmetric quasiextension, then $\rho$ does not violate a Bell inequality with $s_{b}$ settings for Bob and any number of settings for Alice.

Remark: The theorem also holds when Alice and Bob are interchanged.

Proof: The intuition behind this theorem relies on the fact that there are no violations of Bell inequalities when one party has only one measurement setting, thus suggesting that it is unnecessary to extend to copies of Alice's space as well as Bob's. Here is the local hidden variable model that we construct from a quasiextension $H_{\rho}$, on $\mathcal{H}_{A} \otimes \mathcal{H}_{B}^{\otimes s_{b}}$. We set

$$
p_{\mathbf{m}, \mathbf{n}}\left(\left\{E_{i j}^{A}, E_{k l}^{B}\right\}, \rho\right)=\frac{\prod_{i^{\prime}=1}^{s_{a}}\left(\operatorname{Tr} E_{i^{\prime} m_{i^{\prime}}}^{A} \otimes \mathbf{E}_{\mathbf{n}}^{B} H_{\rho}\right)}{\left(\operatorname{Tr} I_{A} \otimes E_{\mathbf{n}}^{B} H_{\rho}\right)^{s_{a}-1}} .
$$

Each $p_{\mathbf{m}, \mathbf{n}}$ is non-negative since $H_{\rho}$ is an entanglement witness. We can substitute this expression in Eq. (4) and verify that we obtain the correct probabilities $P_{i j, k l}(\rho)$ by using the definition of the $B$ vectors, the normalization of the POVMs, and the symmetry of $H_{\rho}$ as before.

This method for constructing LHV theories may be implemented both numerically and analytically. Let us first show a simple analytic construction of a $(2,2)$-symmetric extension for any bound entangled state based on a real unextendible product basis [7]. Let $P_{B E}=$ $I-\sum_{i}\left|a_{i}, b_{i}\right\rangle\left\langle a_{i}, b_{i}\right|$ be the projector onto such a bound entangled state, where $\left\{\left|a_{i}, b_{i}\right\rangle=\left|a_{i}^{*}, b_{i}^{*}\right\rangle\right\}$ is the real unextendable product basis. Our (unnormalized) extension will be $|\Psi\rangle_{A_{2} A_{1}} \otimes|\Psi\rangle_{B_{1} B_{2}}-\sum_{i}\left|a_{i}, a_{i}, b_{i}, b_{i}\right\rangle_{A_{2} A_{1} B_{1} B_{2}}$, where $|\Psi\rangle=\sum_{i}|i i\rangle$. It is evident that this extension has the desired symmetry property. It is not hard to verify that by tracing over the systems $A_{2}$ and $B_{2}$ we obtain $P_{B E}^{2}=P_{B E}$. The existence of a symmetric $(2,2)$ extension implies the existence of both $(2,1)$ and $(1,2)$ symmetric extensions for the state by tracing out copies of $A$ or $B$, so any Bell inequality violation for this class of states must involve more than two measurement settings for both parties.

We have implemented numerical tests for the conditions of these two theorems. First, we look for the existence of a symmetric extension with $H_{\rho} \geq 0$. If such an extension does not exist, there is still the possibility that some other kind of quasiextension does exist. We have focused on the existence of a decomposable entanglement witness $H_{\rho}$ because in both these cases the numerical problem corresponds to a semidefinite program [13]. We label the partitions of $\mathcal{H}_{A}^{\otimes s_{a}} \otimes \mathcal{H}_{B}^{\otimes S_{b}}$ into bipartite systems by $p$ and we denote partial transposition with respect to one of the two subsystems as $T_{p}$. A decomposable entanglement witness may then be written as $H_{\rho}=P+$ $\sum_{p} Q_{p}^{T_{p}}$, where $P \geq 0, Q_{p} \geq 0$ for all $p$.

Semidefinite programs correspond to optimizations of linear functions on positive matrices subject to trace constraints. They are convex optimizations and are particularly tractable both analytically and numerically. We show how to numerically construct symmetric extensions, the decomposable quasiextension case is very similar. The condition that the partial trace of $H_{\rho}$ is $\rho$ is equivalent to requiring that $\operatorname{Tr}(X \otimes \mathbf{I}) H_{\rho}=\operatorname{Tr} X \rho$ for all operators $X$ on $\mathcal{H}_{A} \otimes \mathcal{H}_{B}$. If we write $X$ in terms of a basis $\left\{\sigma_{i}\right\}$ for the real vector space of Hermitian operators, then by linearity it is enough to check that this trace constraint holds for each element of the basis. We assume that the basis is orthogonal in the trace inner product $\operatorname{Tr} \sigma_{i} \sigma_{j}=\delta_{i j}$ and that $\sigma_{0}=I_{d_{A}} \otimes I_{d_{B}} / \sqrt{d_{A} d_{B}}$. The index $i$ ranges from zero to $\left(d_{A} d_{B}\right)^{2}-1$. Consider then this semidefinite program

$$
\begin{aligned}
& \text { minimize } \operatorname{Tr} K \text {, } \\
& \text { subject to } \quad \operatorname{TrSym}_{A} \otimes \operatorname{Sym}_{B}\left(\sigma_{i} \otimes \mathbf{I}\right) K=r_{i}, \quad i>0, \\
& K \geq 0 \text {, }
\end{aligned}
$$

where $r_{i}=\operatorname{Tr} \sigma_{i} \rho$. If the optimum is less than or equal to one, then, by adding a multiple of the identity to the optimal $K$, we obtain some $K_{\rho}$ that satisfies $\operatorname{Tr} K_{\rho}=1$ as well as the other constraints. If we define $H_{\rho} \equiv \mathrm{Sym}_{A} \otimes$ $\operatorname{Sym}_{B}\left(K_{\rho}\right)$ it is clear that $H_{\rho}$ is a $\left(s_{a}, s_{b}\right)$-symmetric extension of $\rho$. Duality properties of semidefinite programs imply that an optimum greater than one precludes the existence of a $\left(s_{a}, s_{b}\right)$-symmetric extension [13].

We have implemented this semidefinite program using SeDuMi [14] for several examples of bound entangled states with $d_{A}=d_{B}=3$. The results are summarized in Table I. For example, the Choi-Horodecki $(\mathrm{C}-\mathrm{H})$ states considered in Ref. [15] depend on a parameter $\alpha$ and include separable $(\alpha \in[2,3]$ ), bound entangled ( $\alpha \in$ $(3,4])$, and nonpositive partial transpose (NPT) states for $\alpha>4$. They turn out to have $(2,1)$-symmetric extensions well into the range for which the states are entangled and even NPT. Over the range $\alpha \in[4.34,4.84]$ they have decomposable symmetric quasiextensions but no symmetric extensions showing that the former property provides a strictly stronger sufficient condition for the existence of a LHV theory. The bound entangled states of [16] do not even have $(1,2)$ extensions. These and many of the Bruß-Peres states [17] are therefore the strongest candidates for bound entangled states with Bell inequality violations. However, we have looked for but did not find Bell inequality violations for $\left(s_{a}=2, s_{b}=2\right)$ settings and three outcome projective measurements and for $(3,3)$ settings and two outcome projective measurements.

Finally we considered Werner states [2] defined in dimensions $d=d_{A}=d_{B} \geq 2$ as $\rho_{W}=\frac{1}{d^{3}-d}[I(d-\Phi)+$ $(d \Phi-1) V]$, where $V$ is the flip operator. Werner [2] 
TABLE I. Numerical results on the existence of symmetric extensions (ext) and decomposable quasiextensions (q-ext) for $\left(s_{a}=1, s_{b}=2\right),\left(s_{a}=2, s_{b}=1\right),\left(s_{a}=1, s_{b}=3\right)$, and $\left(s_{a}=3\right.$, $\left.s_{b}=1\right)$. For the one and two dimensional families of states $[2,15,16]$ we performed a systematic search of the parameter space. For the high dimensional families [17,18] we make qualitative statements based on randomly chosen examples. Note that states may have an $(s, 1)$ extension and no $(1, s)$ extension. We have performed both tests in all cases, but the general results are unaffected although we do find examples of Bruß-Peres states with $(2,1)$ extensions, say, but no $(1,2)$ extensions.

\begin{tabular}{cccc}
\hline \hline & \multicolumn{2}{c}{$(2,1),(1,2)$} & $(3,1),(1,3)$ \\
& ext & q-ext & ext \\
\hline C-H [15]: $\alpha \in$ & {$[2,4.33]$} & {$[2,4.84]$} & {$[2,4.00]$} \\
Complex UPB [18] & Yes & Yes & Few \\
H-L [16] & No & No & No \\
Bruß-Peres [17] & Few & Few & No \\
Werner [2] & $d \geq 3$ & $d \geq 3$ & $d \geq 4$ \\
Werner $d=2, \Phi \geq$ & $-1 / 2$ & $-1 / 2$ & $-1 / 3$ \\
\hline \hline
\end{tabular}

showed that for $\Phi \geq-1+\frac{d+1}{d^{2}}$ these states do not violate any Bell inequality with an arbitrary number $s_{a}, s_{b}$ of von Neumann measurements (in Ref. [19] the author constructs LHV models for arbitrary POVM settings for a more restricted range of $\Phi$ ). We found that using symmetry techniques similar to those in Ref. [20] it is possible to analytically solve the dual optimization problem to the semidefinite program described above; see [21]. The value of the optimum establishes that all Werner states have symmetric extensions so long as $s_{a}+s_{b} \leq d$. Hence these states have LHV theories for all Bell experiments where the minimum number of settings $s=\min \left(s_{a}, s_{b}\right)$ satisfies $s+1 \leq d$. This result is more general than Werner's in the sense that, as in Ref. [19], it holds for general POVM elements. It is weaker in the sense that the number of settings is bounded by the dimension of the space. Numerical and analytical results (see Table I and [21]) show that Werner states for $d=2$ actually have symmetric (quasi-) extensions beyond this analytically derived bound.

Even though our method is the most powerful tool to date for constructing local hidden variable theories, we believe that it is unlikely that every LHV model can be constructed from a symmetric quasiextension. It has been proven that only separable states have $\left(s_{a}=1, s_{b} \rightarrow \infty\right)$ extensions; see Refs. [10,22]. This result can be extended to quasiextensions [23] even though LHV theories for entangled states with an arbitrary number of settings do exist $[2,19]$. Our work is only the starting point for a more thorough exploration of the existence of LHV models and (quasi-) extensions for entangled quantum states.

We thank Dave Bacon and Ben Toner for providing us with extremal Bell inequalities used in some of the numerical work. We are very grateful to Michael Wolf for his insightful comments on the original draft of this paper and for bringing Ref. [10] to our attention. B. M.T. and A.C.D. acknowledge support from the NSF under Grant No. EIA-0086038. A. C. D. acknowledges support from the Caltech MURI Center for Quantum Networks administered by the ARO under Grant DAAD19-00-10374 and B.M.T. from the NSA and the Advanced Research and Development Activity through ARO Contract No. DAAD19-01-C-0056. A. C. D. thanks Mark Kasevich and Steve Girvin for their hospitality at Yale University where part of this work was completed. D. S. thanks the IQI for support.

[1] J. Bell, Physics (Long Island City, N.Y.) 1, 195 (1964).

[2] R. Werner, Phys. Rev. A 40, 4277 (1989).

[3] A. Peres, Found. Phys. 29, 589 (1999).

[4] M. Horodecki, P. Horodecki, and R. Horodecki, Phys. Rev. Lett. 80, 5239 (1998).

[5] R. Werner and M. Wolf, Phys. Rev. A 61, 062102 (2000); 64, 032112 (2001); W. Dür, Phys. Rev. Lett. 87, 230402 (2001); A. Acin, T. Durt, N. Gisin, and J. Latorre, Phys. Rev. A 65, 052325 (2002); A. Acin, V. Scarani, and M. Wolf, quant-ph/0206084; D. Kaszilikowski, M. Zukowski, and P. Gnacinski, quant-ph/0107154.

[6] I. Pitowsky, Math. Progr. 50, 395 (1991).

[7] C. Bennett et al., Phys. Rev. Lett. 82, 5385 (1999).

[8] R. F. Werner and M. M. Wolf, Quantum Inf. Comput. 1, 1 (2001).

[9] A connection between Bell inequalities and extensions has been made previously by R. Werner [10].

[10] R. F. Werner, Lett. Math. Phys. 17, 359 (1989).

[11] A. C. Doherty, P. A. Parrilo, and F. M. Spedalieri, Phys. Rev. Lett. 88, 187904 (2002).

[12] M. M. Wolf (private communication).

[13] L. Vandenberghe and S. Boyd, SIAM Rev. 38, 49 (1996).

[14] J. Sturm, SEDUMI VERSION 1.05,2001, http://fewcal.kub.nl/ sturm/software/sedumi.html.

[15] P. Horodecki, M. Horodecki, and R. Horodecki, Phys. Rev. Lett. 82, 1056 (1999).

[16] P. Horodecki and M. Lewenstein, Phys. Rev. Lett. 85, 2657 (2000).

[17] D. Bruß and A. Peres, Phys. Rev. A 61, 30301(R) (2000).

[18] D. DiVincenzo et al., quant-ph/9908070.

[19] J. Barrett, Phys. Rev. A 65, 042302 (2002).

[20] T. Eggeling and R. F. Werner, Phys. Rev. A 63, 042111 (2001).

[21] B. M. Terhal, A. C. Doherty, and D. Schwab, quant-ph/ 0210053.

[22] B. Schumacher and R. Werner (private communication); M. Fannes, J. Lewis, and A. Verbeure, Lett. Math. Phys. 15, 255 (1988); G. Raggio and R. F. Werner, Helv. Phys. Acta 62, 980 (1989).

[23] F. Spedalieri, A. C. Doherty, and P. Parrilo (to be published). 